Charry, O., Tamayo, C., \& Jaramillo, D. (2020). ¿Matemáticas para todos? Deconstrucciones desde un territorio chocoano. Revista Latinoamericana de Etnomatemática, 13(4), 95-119. DOI:

$10.22267 /$ relatem.20134.74

Artículo recibido el 1 de noviembre de 2020. Aceptado para publicación el 9 de diciembre de 2020

\title{
¿Matemáticas para todos? Deconstrucciones desde un territorio chocoano
}

\author{
Mathematics for all? Deconstructions from a Chocoano territory
}

\author{
Óscar Charry ${ }^{1}$ \\ Carolina Tamayo ${ }^{2}$ \\ Diana Jaramillo ${ }^{3}$
}

\begin{abstract}
Resumen
En este documento se presentan algunos avances de una investigación doctoral que se viene desarrollando con maestros y maestras de la Institución Educativa Katío Chamí del municipio de El Carmen de Atrato (Chocó, Colombia). El propósito de este artículo es realizar una problematización del enunciado "matemáticas para todos" en el campo del currículo de matemáticas. Enunciado que se torna problemático cuando, en términos curriculares, se alimenta una imagen disciplinar de la matemática. Esa problematización es orientada por una actitud terapéutico deconstruccionista inspirada en las formas de pensar y practicar la filosofía de Ludwig Wittgenstein y Jacques Derrida. Apoyados en la perspectiva política cultural de la educación matemática, en interlocución con algunos autores que fundamentan sus estudios en las teorizaciones foucaultianas, buscamos desnaturalizar el enunciado "matemáticas para todos", es decir, mostrar que él no es natural ni inevitable, sino que es una construcción discursiva que promueve simultáneamente procesos de inclusión y exclusión de individuos, pueblos y hasta naciones. Al mirar las matemáticas desde una perspectiva indisciplinar, apoyados en las contribuciones del filósofo Ludwig Wittgenstein, logramos ver el enunciado "matemática para todos" de otras maneras. Ver tal enunciado, ya no como una agenda política que promueve la homogeneización de la sociedad, sino como una agenda política de lucha y resistencia por la preservación de diferentes formas de vida, por la recuperación de aquellos sujetos que han sido históricamente excluidos a través de relaciones asimétricas de poder, por ejemplo, indígenas.
\end{abstract}

Palabras clave: Perspectiva Indisciplinar; Perspectiva Wittgensteiniana; Terapia Deconstruccionista; Teorizaciones Foucaultianas; Currículo de Matemáticas.

\footnotetext{
Abstract

This paper presents some advances of a doctoral research that is in its third year of development. The purpose of this paper is to make a problematization of the statement "mathematics for all" in the field of the mathematics curriculum. Statement that becomes problematic when, in curricular terms, a disciplinary image of mathematics is fed. This problematization is guided by a deconstructionist therapeutic attitude inspired by the ways of thinking and practicing the philosophy of Ludwig Wittgenstein and Jacques Derrida. Supported by the cultural

${ }^{1}$ Doctorando en educación de la Facultad de educación de la Universidad de Antioquia (Medellín, Colombia). Profesor de la Facultad de Educación de la Universidad de Antioquia (Medellín, Colombia). E-mail: oscar.charry@udea.edu.co

${ }^{2}$ Doctora en educación por la Universidad Estadual de Campinas-UNICAMP (Campinas, São Paulo, Brasil). Profesora de la Facultad de Educación de la Universidad Federal de Minas Gerais (Belo Horizonte, Brasil). Profesora de la Facultad de Educación de la Universidad de Antioquia (Medellín, Colombia). E-mail: carolinatamayo@ufmg.br; carolina.tamayoo@udea.edu.co.

${ }^{3}$ Doctora en educación por la Universidad Estadual de Campinas-UNICAMP (Campinas, São Paulo, Brasil). Profesora de la Facultad de Educación en la Universidad de Antioquia (Medellín, Colombia). E-mail: diana.jaramillo@udea.edu.co.
} 
political perspective of mathematics education, we seek to denature the statement "mathematics for all", that is, to show that it is neither natural nor inevitable, but rather a discursive construction that simultaneously promotes processes of inclusion and exclusion of individuals, peoples and even nations. Looking at [mathematics] from an undisciplined perspective, supported by the contributions of the philosopher Ludwig Wittgenstein, we are able to see the statement "[mathematics] for everyone" in other ways. Looking at that statement no longer as a political agenda that promotes the homogenization of society, but as a political agenda of struggle and resistance for the preservation of different ways of life, for the recovery of those subjects who have been historically excluded through asymmetric power relations, for example, indigenous people.

Keywords: Undisciplined Perspective; Wittgensteinian Perspective; Deconstructionist Therapy; Foucauldian Studies; Mathematics Curriculum.

\section{PRESENTACIÓN}

Este artículo es derivado de una investigación doctoral ${ }^{4}$ titulada "currículo [de matemáticas $]^{5}$ : deconstrucción terapéutica ${ }^{6}$ con maestros y maestras de la Institución Educativa Katío Chamî?". El objetivo de esa tesis doctoral es "deconstruir terapéuticamente el currículo [de matemáticas], disciplinarmente organizado, a partir de una problematización indisciplinar de prácticas sociales". Para conducir metodológicamente esa investigación, se asume una actitud terapéutico deconstruccionista inspirada en los trabajos de los filósofos Ludwig Wittgenstein y Jacques Derrida.

El trabajo de campo se viene desarrollando con maestras y maestros de la Institución Educativa Katío Chamí de la comunidad indígena de Sabaleta del municipio de El Carmen de Atrato (Chocó, Colombia). Esa Institución Educativa (IE), que atiende principalmente a población indígena, cuenta con maestras y maestros indígenas de la comunidad de Sabaleta, y maestras y maestros afrodescendientes provenientes del interior del departamento del Chocó.

\footnotetext{
${ }^{4}$ Investigación financiada durante el año 2018 por el proyecto "Jóvenes Excelentes y Líderes del Nuevo Chocó”; y a partir del año 2020, por el programa de Excelencia Doctoral del Bicentenario - Minciencias.

${ }^{5}$ Más adelante se explicará el uso de los corchetes.

${ }^{6}$ Las palabras en itálica hacen parte de la perspectiva filosófica a la cual nos aproximamos en este documento, tienen un significado propio en el pensamiento Embera o corresponden a las voces de los maestros y maestras de la IE Katío Chamí.

${ }^{7}$ De acuerdo con Mecha (2015, p. 6), “[...] en los Embera se presentan diferencias, a partir del medio en el que viven, dividiéndose en los Embera Eyapida, gente de montaña, Embera Oipida, gente de selva y Embera Topida, gente de río. Dentro de los primeros se encuentran los Embera Katío y los Chamí". En esta comunidad indígena habitan Embera Chamí. El nombre de la Institución Educativa incluye "Katío", pues inicialmente se buscaba atender la población estudiantil de las comunidades Embera Katíos cercanas a la institución. Sin embargo, estos grupos crearon sus propias instituciones.
} 
Charry, O., Tamayo, C., \& Jaramillo, D. (2020). ¿Matemáticas para todos? Deconstrucciones desde un territorio chocoano. Revista Latinoamericana de Etnomatemática, 13(4), 95-119. DOI:

$10.22267 /$ relatem.20134.74

Algunas de las voces traídas, desde la IE, para dialogar y reflexionar en este artículo corresponden a la del maestro de la sección de preescolar, quien es también el Gobernador ${ }^{8}$ de la comunidad desde el año 2019; a la del maestro de primaria, quien es el encargado del área de matemáticas en la sección de primaria (estos dos maestros son Embera Chamí); a la del Rector de la IE; y, a la de la maestra de bachillerato, quien está encargada del área de matemáticas para esa sección (ambos de origen afrodescendiente). Las voces de estos maestros emergieron en el marco de las entrevistas ${ }^{9}$ y los encuentros reflexivos ${ }^{10}$ que se tuvieron en la IE como parte del trabajo de campo. Esas voces se ponen en diálogo con las de algunos maestros y líderes indígenas de los departamentos de Chocó y de Antioquia.

En este documento proponemos una problematización del enunciado "matemáticas para todos" en el campo del currículo de matemáticas. Enunciado que se torna problemático cuando se alimenta, en términos curriculares, una imagen disciplinar de la matemática. Así, en primer lugar, presentamos algunos aspectos metodológicos y teóricos que orientan esa investigación. Luego, realizamos una problematización del enunciado "matemáticas para todos" a partir de las voces de los protagonistas de la investigación doctoral, en diálogo con voces de otros maestros y líderes indígenas colombianos. Finalmente, miramos las matemáticas desde una perspectiva indisciplinar, apoyados en las contribuciones del filósofo Ludwig Wittgenstein, para tratar de ver el enunciado "matemática para todos" de otras maneras.

\section{ASPECTOS METODOLÓGICOS Y TEÓRICOS}

El camino metodológico que asumimos en la investigación antes mencionada es la terapia deconstruccionista, inspirada en la terapia filosófica de Ludwig Wittgenstein, en interlocución con la deconstrucción de Jacques Derrida. Esa terapia deconstruccionista es considerada como una actitud y no como un método genérico que sigue unas reglas fijas

\footnotetext{
${ }^{8}$ De acuerdo con la Constitución Política de Colombia del año 1991, las entidades territoriales, como es el caso de los territorios indígenas (Art. 286), "gozan de autonomía para la gestión de sus intereses, y dentro de los límites de la Constitución y la ley" (Art. 287). En ese sentido, las comunidades indígenas tienen derecho a ser gobernadas por sus propias autoridades; una de ellas, es el Gobernador.

${ }^{9}$ Las entrevistas que son citadas en este documento fueron concedidas a Óscar Charry en el marco del trabajo de campo de la tesis doctoral ya referida.

${ }^{10}$ Los encuentros reflexivos son espacios donde se reúnen los maestros y directivos de la IE Katío Chamí para discutir diferentes asuntos relacionadas con la educación en su comunidad. El investigador Óscar Charry viene haciendo parte de estos encuentros en el marco de su trabajo de campo.
} 
(Miguel, 2015). Desde esa perspectiva, se problematiza la creencia de que es posible definir un método de investigación universalmente válido, o un método generalizable a una serie de problemas. En ese sentido, dice Wittgenstein (1995): "no hay un método, sino que hay en Filosofía de hecho, métodos, tal como hay diversas terapias" (\$133).

La terapia deconstruccionista, de acuerdo con Miguel (2015), intenta abandonar el deseo permanente de explicar estableciendo relaciones de causa y efecto. Pues, como dice Wittgenstein (2007):

(...) la realización de una explicación ya es defectuosa, porque uno solo tiene que organizar correctamente lo que sabe, y no agregar nada, y la satisfacción a la que aspira la explicación viene por sí misma (...). La explicación es, comparada con la impresión que la descripción causa, demasiado insegura. (p. 194).

La terapia deconstruccionista también intenta evitar el constante deseo de teorizar, de establecer generalizaciones. En términos de Wittgenstein (1995): "correcto fue no dejar que nuestra investigación fuese una investigación científica. (...). Y no debemos producir ninguna especie de teoría. (...). Toda la explicación tiene que acabar y ser substituida apenas por la descripción.” (\$109).

Una actitud terapéutico deconstruccionista posibilita situarnos como investigadores en las prácticas sociales, y describirlas tal y como ellas se nos presentan (Miguel, 2015). Prácticas sociales que son entendidas como "[...] un conjunto regulado de acciones intencionales efectivas que movilizan simultáneamente conocimiento, propósitos, deseos, recuerdos, afectos, valores, poderes, etc., que son escenificadas conjuntamente por humanos y otros seres naturales [...]" (Souza y Miguel, 2020, p. 171).

Al situarnos en las prácticas sociales, es posible problematizar las relaciones asimétricas de poder que se establecen en los debates interculturales, buscando preservar la diversidad cultural (Tamayo y Cuellar, 2016). Cuando usamos la palabra preservación, lo hacemos siguiendo a esos mismos autores, en el sentido de mantener un "diálogo intercultural poscolonial, es decir, un diálogo que no tenga como base la imposición unilateral de valores, formas de ver y pensar de una cultura para otra” (p. 54).

Esa descripción de prácticas sociales no es un relato, ni tampoco procede a través del establecimiento de relaciones causales; ella es una descripción problematizadora que procede 
Charry, O., Tamayo, C., \& Jaramillo, D. (2020). ¿Matemáticas para todos? Deconstrucciones desde un territorio chocoano. Revista Latinoamericana de Etnomatemática, 13(4), 95-119. DOI:

$10.22267 /$ relatem.20134.74

a través del establecimiento de relaciones analógicas o semejanzas de familia entre diferentes juegos de lenguaje (Miguel, 2015). Desde esta perspectiva, según este mismo autor, las prácticas sociales son vistas como juegos de lenguaje normativos, es decir, como escenificaciones simbólico-corporales de un conjunto de acciones orientadas por reglas. Así, el lenguaje se practica con todo el cuerpo y "no sólo con la vibración culturalmente regulada de los sonidos emitidos por nuestras cuerdas vocales” (Miguel, 2016, p. 372). Como dice Wittgenstein (1995): un juego de lenguaje es el "(...) todo formado por el lenguaje con las actividades con las cuales él está entrelazado" (§7).

Los juegos de lenguaje presentan parecidos, que Wittgenstein (1995) denomina "semejanzas de familia; porque las diversas semejanzas entre los miembros de una familia, constitución, rasgos faciales, color de los ojos, caminar, temperamentos, etc., etc., se sobreponen y se cruzan de la misma manera. (...)” (§ 67). Con la noción semejanzas de familia este filósofo abandona la idea de una esencia metafísica en los juegos de lenguaje, al posibilitar el establecimiento de analogías que "muestran que no hay necesariamente algo común a todos los juegos de lenguaje" (Condé, 2004, p. 55). En otras palabras, utilizando la metáfora de la cuerda de Glock (1997), los juegos de lenguaje no los conecta un único hilo, "sino, por así decirlo, una sobreposición de diferentes fibras, como en una cuerda" (p. 325).

El objetivo de la terapia deconstruccionista es realizar una presentación panorámica del uso de nuestro lenguaje (Souza y Miguel, 2020). La visión panorámica es fundamental para Wittgenstein (2007, p. 200), ya que ella marca “(...) la forma como nosotros vemos las cosas, [como] una especie de 'visión del mundo' (...)”. Según Wittgenstein (1995),

(...) una de las fuentes principales de incomprensión reside en el hecho de que no tenemos una visión panorámica del uso de nuestras palabras. Nuestra gramática no se deja ver panorámicamente. - La presentación panorámica facilita la comprensión, la cual de hecho consiste en <<nosotros ver las conexiones〉>. De ahí la importancia de encontrarse y de inventarse los términos intermedios (\$122).

En ese sentido, Miguel (2015) encuentra articulaciones entre los filósofos Ludwig Wittgenstein y Jacques Derrida para pensar la investigación en el campo de la Educación Matemática. Articulaciones que él ha denominado terapia deconstruccionista que, como ya dijimos, está también inspirada en la deconstrucción derridiana. Esa deconstrucción derridiana, según Skliar (2005), significa deshacer, sin pretender destruir, un pensamiento que se muestra dominante. Para este autor, la deconstrucción es un "acto de justicia” (p. 21), 
que no acontece sin amorosidad. Así lo expresa Jacques Derrida en su diálogo con Élisabeth Roudinesco:

(...) la experiencia de la "deconstrucción” nunca ocurre, eso sí, sin amor (...) siempre hay un momento en que declara, con la mayor sinceridad, la deuda, el reconocimiento y la necesidad de ser fiel a la herencia para reinterpretarla y reafirmarla interminablemente. (Derrida y Roudinesco, 2004, p. 13).

Esto quiere decir, como lo señala Élisabeth Roudinesco, que "la mejor manera de ser fiel a una herencia es serle infiel, es decir, no recibirla literalmente, como una totalidad, sino más bien pescarla en falta, captar su "momento dogmático" (...)” (Derrida y Roudinesco, 2004, p. 10).

Consideramos que a partir de una actitud terapéutico deconstruccionista es posible rescatar aquellos sujetos que han sido históricamente excluidos a través de relaciones asimétricas de poder, como es el caso de algunos indígenas. Sujetos que, según Condé (2004), no se pueden concebir fuera del lenguaje, fuera de una forma de vida. Forma de vida que es entendida como “(...) el entrelazamiento entre cultura, visión de mundo y lenguaje” (Glock, 1997, p. 174).

Así, la actitud terapéutico deconstruccionista también es una actitud ética, política, de lucha y de resistencia por la preservación de comunidades indígenas, como la de Sabaleta. Esta actitud para investigar viene siendo practicada en Colombia en la Educación Matemática por autores como Tamayo (2017), Quiceno y Montoya (2020), Charry y Jaramillo (2020), Charry, Tamayo y Jaramillo (2020), y Charry, Jaramillo y Tamayo (2020).

En este artículo, también encontramos algunas articulaciones con los estudios foucaultianos traídos al campo del currículo por Veiga-Neto (2001) y con la perspectiva política cultural de la educación matemática (Valero, Andrade-Molina y Montecino, 2015). Desde esa última perspectiva, se problematiza las formas cómo el currículo de matemáticas, “en un tiempo y espacio específicos, moldea las direcciones en las cuales nos convertimos en un determinado sujeto en relación con el conocimiento matemático, conforme éste es configurado en las prácticas pedagógicas dentro de la escuela como institución social” (Valero, 2018, p. 47).

\section{3. ¿"MATEMÁTICAS PARA TODOS"?}

El Proyecto Educativo Comunitario (PEC) de la IE Katío Chamí se elaboró “en el marco de la normatividad establecida por el MEN [Ministerio de Educación Nacional] (...) para 
Charry, O., Tamayo, C., \& Jaramillo, D. (2020). ¿Matemáticas para todos? Deconstrucciones desde un territorio chocoano. Revista Latinoamericana de Etnomatemática, 13(4), 95-119. DOI:

10.22267/relatem.20134.74

responder a las oportunidades sociales, económicas y culturales del entorno y contexto (...)" (PEC, 2015, p. 8). La propuesta curricular de esa IE promueve "la formación de seres humanos con un perfil basado en competencias básicas, ciudadanas y específicas que le permitan el ingreso al mundo laboral o la educación superior" (Ibídem, p. 20). Esa propuesta busca "fomentar el pensamiento emprendedor, la creatividad y la innovación como factores que faciliten el ingreso de los estudiantes en los procesos sociales, económicos y culturales (...) [para] su propio desarrollo, el de su región y el de nuestro país" (Ídem). La formación en competencias se considera importante, pues posibilita que los estudiantes pongan "en práctica el conocimiento mediante la solución a problemas de la vida cotidiana, del entorno y contexto" (PEC, 2015, p. 21).

En el marco de esa propuesta curricular, el docente de la IE se encargaría de la selección de "los estándares con los cuales va a organizar la clase (...)" (Ibídem, p. 22). También, el docente debe apropiarse "de los resultados obtenidos en las pruebas SABER ${ }^{11}$ para replantear y orientar su práctica pedagógica" (PEC, 2015, p. 13). Se espera que ellos realicen "evaluaciones a sus estudiantes estilo prueba saber" (Ídem). Esto, pues uno de los requisitos para graduarse de esta IE, es que los estudiantes de grado undécimo presenten las Pruebas Saber 11 (PEC, 2015).

El discurso anterior, promovido por el Ministerio de Educación Nacional (MEN), atiende a un modelo curricular por competencias que se viene desarrollando desde la década del 90 en Colombia, bajo la consigna rectora "educación para todos" (Mejía, 2004). En esa década, según este autor, se produjo un conjunto de reformas educativas que promueve para la escuela "un proyecto productivista en el cual las competencias se construyen en función de un mercado globalizado y el criterio de este no sólo comienza a orientar los fines de la educación, sino también la dinámica interna de las instituciones" (p. 6). Esa propuesta curricular por competencias define unos "mínimos requeridos para vivir en el conocimiento de la sociedad globalizada (estándares) y deriva de ellos los "saber hacer" para la empleabilidad que genera un mundo en una crisis profunda de trabajo y empleo (competencias)" (Ibídem, p. 8). En el marco de esa propuesta curricular, continuando con

\footnotetext{
${ }^{11}$ Las Pruebas Saber son evaluaciones estandarizadas que se realizan en todo Colombia a los estudiantes de grado 11, que corresponde al último año escolar en las instituciones de enseñanza media.
} 
Mejía (2004), los estándares y competencias deben ser evaluados por medio de pruebas estandarizadas, como es el caso del Examen o Prueba Saber 11.

Con respecto a las Pruebas Saber 11, la maestra de bachillerato afirma que cuando los estudiantes ingresan a grado once, desde principio de año los maestros inician "el proceso para irlos preparando para que se enfrenten a [esa prueba]"12. La manera como realizan ese proceso de preparación, en palabras de la maestra, es la siguiente: “[trabajamos] con cartillas [tipo prueba Saber] (...), contextualizándolas de acuerdo con el medio en el cual nosotros nos encontramos (...). Trabajamos paso a paso para que ellos [se puedan desempeñar bien] cuando les toque presentar el examen"13.

Sin embargo, como señala esta misma maestra, a pesar del esfuerzo que realizan los maestros de grado once, hasta el momento "no han obtenido ["buenos"] resultados, porque los muchachos tienen dificultades en la comprensión lectora y en el proceso de las operaciones" 14 . Esa situación hace que ella, como maestra de matemáticas, reflexione sobre su labor. Dicho en sus propias palabras: "uno se siente desanimado, y se pregunta si será que no está haciendo el proceso bien, porque nosotros damos lo más que podemos (...)"15.

En la voz de esta maestra se refleja el deseo del maestro de matemáticas de que sus estudiantes obtengan "buenos" resultados. Como dice ella: "como todo docente, el sueño de uno es que nuestros estudiantes entiendan, comprendan la temática que uno trae"16. Para el Gobernador de Sabaleta también es importante que los niños, las niñas y los jóvenes de su comunidad aprendan matemáticas pues, según él, “los números son primordiales para contar, para conocer, en la vida, porque [los jóvenes más adelante] van a manejar su propia economía"17.

Sin embargo, para el Gobernador también es importante que los niños, niñas y jóvenes aprendan los conocimientos propios de su comunidad, por eso vienen trabajando en la recuperación de sus propias matemáticas; que él denomina "Mate-Chamí, que es matemática

\footnotetext{
12 Entrevista, 31 de octubre de 2019.

${ }^{13}$ Entrevista, 31 de octubre de 2019.

${ }^{14}$ Entrevista, 31 de octubre de 2019.

${ }^{15}$ Entrevista, 31 de octubre de 2019.

${ }^{16}$ Entrevista, 31 de octubre de 2019.

${ }^{17}$ Entrevista, 24 de octubre de 2019.
} 
Charry, O., Tamayo, C., \& Jaramillo, D. (2020). ¿Matemáticas para todos? Deconstrucciones desde un territorio chocoano. Revista Latinoamericana de Etnomatemática, 13(4), 95-119. DOI:

10.22267/relatem.20134.74

en el mundo Cham $^{\curvearrowright}{ }^{18}$. En esa misma dirección apunta el PEC de la IE Katío Chamí, el cual dice que la propuesta curricular de la IE debe también "fortalecer el territorio, con bienestar social; desde el conocimiento ancestral, a partir de la familia, para el arraigo cultural y social (...) (PEC, 2015, p. 8).

Con relación a la expresión "Mate Chamî", utilizada por el Gobernador de la comunidad indígena de Sabaleta, podríamos decir, apoyados en Monteiro (2005), que existe, en esa denominación una actitud política, de lucha y resistencia por la preservación de los conocimientos ancestrales de la comunidad, al anteponerlos a las matemáticas escolares. Unas matemáticas escolares, entendidas como una transposición escolar de las matemáticas académico-científicas (Miguel, 2016) que, según D’Ambrosio (2008), se han "concebido como la ciencia de los números y de las formas, de las relaciones y de las medidas, de las inferencias, y sus características apuntan hacia la precisión, el rigor y la exactitud" (p. 70). Esas matemáticas se presentan "como un dios más sabio, más milagroso y más poderoso que las divinidades tradicionales y de otras culturas" (Ibídem, p. 71).

Desde la perspectiva de Lizcano (2006), esas matemáticas escolares se entenderían “como el desarrollo de una serie de formalismos característicos de la peculiar manera de entender el mundo de cierta tribu de origen europeo" (p. 190). Una matemática que logró ocultar las supersticiones y prejuicios en que se basaba, y consiguió imponerse como la única matemática posible (Lizcano, 2006).

El aprendizaje de esas matemáticas en nuestra sociedad estaría justificado, según Valero (2017a), en que ellas les otorgan a los individuos cierto tipo de empoderamiento, el cual es intrínseco a las propias matemáticas. Es decir, según esta misma autora, esas matemáticas empoderan "porque hay algo esencial propio del conocimiento matemático en sí que se transfiere a quienes logran apropiarse de él" (106). Valero (2017a) no sólo problematiza ese argumento del empoderamiento intrínseco de las matemáticas, que sirve para justificar su enseñanza, sino también la visión utilitarista de esas matemáticas; es decir, la capacidad que las matemáticas le otorgarían a un individuo para resolver problemas.

\footnotetext{
${ }^{18}$ Entrevista, 24 de octubre de 2019.
} 
Esa visión utilitarista de las matemáticas podría tener algunos efectos indeseables en el contexto de la educación indígena. Esto, porque la importancia de aprender matemáticas está fundamentada, muchas veces, en la necesidad que tienen los indígenas de establecer algunas relaciones comerciales interculturales. Esa necesidad es cuestionada por D'Ambrosio (2008) en los siguientes términos: "sin aprender la "aritmética del blanco", el indio será engañado en sus transacciones comerciales con el blanco. Así como sin cubrir su desnudez y sin dominar la lengua del blanco, el indio difícilmente tendrá acceso a la sociedad dominante" (p. 74).

De manera semejante, el líder indígena Abadio Green Stoce ${ }^{19}$ critica ese discurso utilitarista de las matemáticas dominantes, que él denomina no indígenas, por las consecuencias que éste traería para las comunidades indígenas. Según él:

Las matemáticas no indígenas se han constituido en el derrotero del camino para que la otra cultura [refiriéndose a la occidental] llegue mucho más fácil a nuestras culturas. (...). Con respecto a las matemáticas, se trata de aprenderlas para que los otros que llegan no nos engañen cuando queremos vender algún producto. Para que no nos sigan engañando necesitamos saber, necesitamos saber sumar, restar, multiplicar y dividir. Eso es lo que le han enseñado a los indígenas, creyendo que aprendiendo la lengua castellana y las matemáticas podemos defender la cultura, pero resulta que no. Por privilegiar esos saberes está, detrás, toda una ideología que nos aleja más de la cultura propia, de las tradiciones propias. (Higuita, 2014, p. 43).

En ese sentido, de acuerdo con D’Ambrosio (2008), la defensa de unas matemáticas que deben ser aprendidas por todos, responde a un principio de equidad que va en búsqueda de una sociedad competitiva y excluyente. En palabras de este autor: "esa concepción de equidad conlleva necesariamente la figura del excluido" (p. 68). Al respecto, dice Lizcano (2006):

Todo el proyecto científico, y toda la racionalidad ilustrada (y la política que la acompaña), pueden pensarse como una des-comunal empresa contra las culturas populares y los saberes vernáculos. Desde su origen, hasta nuestros días, en que se ha disfrazado bajo el lenguaje de la modernización y el desarrollo. (p. 196).

Para Valero (2017b), “el impulso de las matemáticas para todos se ha convertido en una verdad naturalizada, un deseo que navega en la sociedad, y que rara vez se problematiza” (p. 119). Podríamos decir que esa declaración “matemáticas para todos" se vuelve problemática

\footnotetext{
${ }^{19}$ Líder indígena de la comunidad Gunadule de Necoclí (Antioquia, Colombia). Su voz es traída en este artículo a partir de la tesis de maestría de Higuita (2014).
} 
Charry, O., Tamayo, C., \& Jaramillo, D. (2020). ¿Matemáticas para todos? Deconstrucciones desde un territorio chocoano. Revista Latinoamericana de Etnomatemática, 13(4), 95-119. DOI:

$10.22267 /$ relatem.20134.74

cuando alimentamos, en términos curriculares, una imagen disciplinar de la matemática. Es decir, cuando entendemos la matemática como un cuerpo unificado, sistematizado y jerarquizado de conocimientos abstractos que son independientes de las prácticas sociales. Es importante aclarar que cuando hablamos de problematizar la declaración "matemáticas para todos" no significa que la estemos negando, o que nos estemos poniendo a favor o en contra de ella. Para nosotros, problematizar ese enunciado significa, inspirados en las contribuciones de Wittgenstein, verlo de otras maneras, tener una visión panorámica de él. Esto, pues, a través de nuestro lenguaje, hemos venido alimentado una imagen disciplinar de la matemática. Una imagen que, como diría Wittgenstein (1995), nos ha mantenido presos, pues "ella misma [está] en nuestro lenguaje, el cual parece repetírnosla implacablemente" (§115). Una imagen, desde la cual, podríamos ver ese enunciado "matemáticas para todos" como la expresión de un deseo "natural" de que "todos" tengan más y mejores "matemáticas".

Sin embargo, esa imagen de la matemática, como dice Vilela (2010) "se presenta como exacta, precisa, verdadera y de resultados únicos" (p. 10). Una matemática que problematiza Lizcano (1992) al decir que sus conocimientos se presentan como "meros productos que brotan de la nada, claros y distintos, (...), meros objetos a perseguir o contemplar en su absoluta identidad, cerrada e inmutable” (p. 2). Esa matemática que se presenta como única y universal, según Lizcano (2006), se ha venido construyendo a través del proceso de borrar las huellas, de borrar los rastros de otras maneras de hacer [matemáticas].

Aquí escribimos la palabra matemáticas entre corchetes, para señalar que es posible verla también desde una perspectiva indisciplinar en el sentido de Miguel (2016). Es decir, es posible, a partir de las contribuciones de Wittgenstein, comprenderla, ya no "como un dominio unificado de conocimientos, sino como un conjunto discreto ilimitado de juegos de lenguaje [normativos] (...)" (Miguel, 2016, p. 332). En otras palabras, como afirma este mismo autor, es posible comprender las [matemáticas] como un conjunto de prácticas sociales.

En ese sentido, como lo dice Santos (2008), no existe una única forma de conocimiento válido, sino tantas formas de conocimiento como prácticas sociales que los generen y los validen; no reconocer esas otras formas de conocimiento, implica deslegitimar las prácticas 
sociales de los que las producen y, de esa manera, fomentar la exclusión social de los que las promueven.

Según Santos (2019), esas otras formas de conocimiento son inseparables de las prácticas sociales; algunas de ellas "surgen y circulan de forma despersonalizada, aunque ciertos individuos en el grupo tengan un acceso privilegiado a esos conocimientos y los formulen con mayor autoridad" (p. 31). Por ejemplo, el Gobernador de la comunidad indígena de Sabaleta dice que los conocimientos ancestrales están en los ancianos y en el Jaibaná (médico espiritual) de la comunidad. Hasta donde él recuerda,

Antes el maestro era el Jaibaná, el Jaibaná era el que sabía todo, y el que lo sabe todavía. Él curaba y cura las enfermedades (...). Los Jaibanás eran los que manejaban la educación. Ellos enseñaban por dónde se podía ir en el territorio, dónde se podía cultivar, dónde no se podía cultivar (...). Esas historias, esas sabidurías, se deben tener en cuenta para nuestra educación. (Entrevista, 24 de octubre de 2019).

De acuerdo con Mecha (2015), para los Embera del Chocó el conocimiento ancestral está en la naturaleza; éste no se queda sólo en el pasado, se actualiza constantemente a través de su uso para enfrentar los problemas cotidianos de los Embera. Este tipo de conocimiento no está fragmentado, categorizado, no se separa de la vida cotidiana; está en sus mentes, en sus cuerpos, en sus corazones (Mecha, 2007). Según este autor, el conocimiento para los Embera no se adquiere de manera individual, sino colectiva, a través de la minga, la charla, la imitación, el juego, etc. El conocimiento para los Embera se crea y se transmite a través de la tradición oral; “(...) de ahí viene su enseñanza de generación en generación, hasta la actualidad" (Mecha, 2007, p. 133). En esa misma dirección, señala Cáisamo (2012) que, en ese proceso de transmitir conocimientos ancestrales, que va también de padres a hijos, "la comunicación oral juega un papel muy importante en la cultura Embera" (p. 170).

Esto nos lleva a problematizar, siguiendo a Knijnik (2006), ese currículo de matemáticas fragmentado, jerarquizado; en el que "la escritura que se practica en las aulas de matemáticas (...) está marcada por el formalismo de los enunciados matemáticos (...)” (p. 153). En el currículo de esas matemáticas, como lo señala esta misma autora, no habría cabida para los conocimientos orales; una oralidad que estaría instituida en otro tipo de racionalidad, en la cual no es posible separar los conocimientos de las prácticas sociales donde se producen y se legitiman. De acuerdo con esta autora, los conocimientos [matemáticos] orales 
Charry, O., Tamayo, C., \& Jaramillo, D. (2020). ¿Matemáticas para todos? Deconstrucciones desde un territorio chocoano. Revista Latinoamericana de Etnomatemática, 13(4), 95-119. DOI:

$10.22267 /$ relatem.20134.74

(...) están fuertemente amalgamados con las prácticas que los producen. Se trata de una relación tan intensamente imbricada que pone en funcionamiento una estrategia opuesta a las presentes en el currículo escolar, una estrategia en la cual la matemática recibe otro significado, muy distinto del que le es atribuido por la Modernidad. (p. 156).

En ese sentido, como lo señala Knijnik (2006), el currículo de matemáticas estaría involucrado en la construcción de subjetividades que se producen en un campo de relaciones de poder y de saber, donde, por lo regular, se termina imponiendo la matemática escolar. Pues, como lo dice Foucault (2002), “(...) no existe un saber sino allí donde se hallan suspendidas las relaciones de poder, (...) el saber no puede desarrollarse sino al margen de sus conminaciones, de sus exigencias y de sus intereses" (p. 28).

Aquí, podríamos decir, apoyados en Valero (2017b), que la declaración "matemáticas para todos", no sólo es la expresión de un deseo de inclusión, sino también un enunciado que promueve simultáneamente procesos de inclusión y de exclusión de individuos, pueblos y naciones de acuerdo con su logro en matemáticas. Ese logro, según esta autora, se ha convertido, en las sociedades actuales, en un indicador del crecimiento económico de las naciones a través de las mediciones estandarizadas. Esas mediciones estandarizadas en la educación, como es el caso de las Pruebas Saber 11, legitiman únicamente los conocimientos disciplinares, tal como lo sugiere el Gobernador de la comunidad de Sabaleta en los siguientes términos:

La verdad, [nosotros] aceptamos [las Pruebas Saber 11] porque es lo que manda el Estado, porque está estipulado bajo una Ley de Educación a nivel nacional y nosotros lo respetamos; que [nuestros jóvenes] conozcan ese mundo, pero también me gustaría que ellos $\left[\mathrm{ICFES}^{20}\right]$ conocieran nuestro mundo, que recopilen nuestra historia, y que esa historia también salga en esas pruebas, pues [esa prueba] sólo trae [asuntos] del mundo occidental. (Entrevista, 24 de octubre de 2019).

Así, la declaración "matemáticas para todos" terminaría generando mecanismos de exclusión de "formas de vida y de ser, que no se asimilan a la deseada" (Valero y Knijnik, 2015, p. 37). Es decir, ese enunciado promueve al mismo tiempo un proceso de exclusión, como lo señalan Valero y García (2014):

Cuando el discurso internacional en educación matemática afirma que las matemáticas son para todos, o los documentos curriculares se refieren a lo que debería lograr toda la población estudiantil de un país, tales enunciados efectúan de por sí una categoría de

\footnotetext{
${ }^{20}$ Instituto Colombiano para la Evaluación de la Educación en Colombia (ICFES).
} 
exclusión de todos aquellos para quienes las matemáticas no son una posibilidad, o todos aquellos que no lograrán lo esperado. (p. 510).

Según esas mismas autoras, a través del currículo de matemáticas se terminan excluyendo las subjetividades no deseadas; aquellas que están relacionadas con formas de vida diferentes, que pudieran llegar a ser incluso irreconciliables con las del sujeto deseado; este último, dotado de las competencias necesarias para contribuir al crecimiento económico de las naciones. Así, de acuerdo con Valero (2017b),

la historización del surgimiento de la declaración 'la matemática es para todos', lo que permite evidenciar es cómo el crecimiento económico, el desarrollo y el progreso se articulan con los logros matemáticos de los niños para la fabricación de un nuevo tipo de ciudadano (...). (p. 130).

Consideramos que ese tipo de sujeto deseado que promueve el enunciado "matemáticas para todos" puede llegar a ser irreconciliable con las formas de vida de algunas comunidades indígenas del departamento del Chocó. Para el líder indígena Cáisamo (2012), "la política de integración [inclusión] del Estado Colombiano, hoy sigue interviniendo en [sus] territorios y comunidades mediante [ese] modelo de desarrollo económico, para mantener el control y expansión territorial (...)” (p. 28). Un modelo de desarrollo que, según Mecha (2015), fomenta la riqueza material a través de la explotación, dominación y domesticación de la naturaleza.

Sin embargo, cómo lo señalan Charry y Jaramillo (2020), algunos pueblos indígenas del Chocó proponen un Plan de Vida que se opone a ese modelo de desarrollo moderno (derivado de la modernidad), y que se teje en una relación horizontal con la naturaleza. Según OREWA (2010), el desarrollo es "bienestar y este se construye hacia el futuro, a partir del pasado" (p.

32). El bienestar social para los indígenas del Chocó

[...] es mantener libre y salva a la Madre Tierra, porque es ella quien permite que los Pueblos indígenas puedan vivir tranquilos, en paz, en armonía con la naturaleza y suministra todo el bienestar social, aquí en este pensamiento no prevalece el recurso económico para encontrar felicidad, los indígenas para el bienestar social no solo piensan en lo humano sino en todos los seres que lo rodea o la existencia de la Madre Tierra (Mecha, 2015, p. 29).

En suma, podríamos decir, apoyados en Veiga-Neto y Lopes (2011), que el enunciado "matemáticas para todos" promueve una idea de "inclusión excluyente", si lo miramos situados en las matemáticas escolares. Según estos autores, “toda acción de inclusión es una 
Charry, O., Tamayo, C., \& Jaramillo, D. (2020). ¿Matemáticas para todos? Deconstrucciones desde un territorio chocoano. Revista Latinoamericana de Etnomatemática, 13(4), 95-119. DOI:

10.22267/relatem.20134.74

acción de dominación, pues presupone traer para el campo de acción de algunos 'aquellos otros' que, históricamente, no pertenecían al campo que fueron de él excluidos" (p. 110). Es decir, desde esa perspectiva, "educar a los otros es traer 'esos otros' para nuestra cultura" (Ídem). La palabra dominación, la utilizan estos mismos autores "para designar simplemente todas y cada una de las acciones en que algunos buscan llevar a otros a su dominio, a su morada" (Veiga-Neto y Lopes, 2012, p. 61). En palabras de Veiga-Neto (2001):

La inclusión puede ser vista como el primer paso en una operación de orden, pues es necesario acercarse al otro, para que se de un primer (re)conocimiento, para que se establezca algún saber, por pequeño que sea, acerca de ese otro. Detectada alguna diferencia, se establece un asombro, seguido de una oposición dicotómica: el mismo no se identifica con el otro, que ahora es un extraño. (p. 27).

Quien lleva la mejor parte en esa operación de orden, según este mismo autor, es quien opera la dicotomía. El objetivo de esa operación de orden es tratar de mantener un tipo de orden en el mundo, a través de las operaciones de "aproximación-conocimiento-diferenciación, o sea, inclusión-saber-exclusión" (Veiga-Neto, 2001, p. 27). Es en ese sentido que decimos que el deseo de inclusión manifestado en el enunciado "matemáticas para todos" promueve una “inclusión excluyente", cuando nos situamos en una imagen disciplinar de la matemática; es decir, cuando vemos la matemática como un cuerpo unificado, sistematizado y jerarquizado de conocimientos abstractos que son independientes de las prácticas sociales.

\section{CURRÍCULO DE “[MATEMÁTICAS] PARA TODOS”}

De acuerdo con el maestro indígena Cuellar ${ }^{21}$ (2017), pensar en una educación indisciplinar, es decir, a partir de prácticas sociales, implica poner en cuestión el hecho de que muchos conocimientos ancestrales no se aprenden en la escuela moderna (derivada de la modernidad), pues "en ella no se cruzan las supuestas fronteras entre culturas, parece que se demarcan con más fuerza las diferencias culturales, es como si no se pudiera conectar esos dos mundos distintos, mundo indígena y mundo occidental” (p. 32).

En esa misma línea, el Gobernador de la comunidad indígena de Sabaleta destaca que la educación indígena debe posibilitar que niños, niñas y jóvenes de la comunidad conozcan, entre otros aspectos, acerca de "cómo fue fundada o cómo fue creada la comunidad,

\footnotetext{
${ }^{21}$ Maestro indígena de la comunidad Gunadule de Necoclí (Antioquia).
} 
[conozcan] la autonomía, el territorio, la cultura y la unidad"22. Para Cuellar (2017), el deber de los maestros indígenas es, desde las escuelas, "transgredir con acciones para continuar practicando la educación que heredamos de nuestros ancestros, y así aprender que los saberes están en las prácticas sociales (...)” (p. 147). De esa manera, la escuela se pone al servicio de las necesidades de la comunidad, procurando que niños, niñas y jóvenes también aprendan a conocer el territorio, las formas de gobierno, la lengua y la ley de origen del pueblo indígena (Cuellar, 2017).

En ese sentido, desde el año 2018, los maestros de la IE Katío Chamí vienen construyendo para la sección de bachillerato una propuesta denominada Tejido de Saberes. Cuenta la maestra de bachillerato que este documento fue presentado por FEDEOREWA para la sección de primaria, y ahora los maestros de bachillerato deben adaptarlo para su sección. Según ella, "FEDEOREWA [les] ha dicho que no lo [llamen] plan de área, sino Tejido de Saberes" ${ }^{23}$. El Tejido de Saberes es un documento vivo (en constante construcción y reflexión), que busca poner en diálogo los conocimientos escolares con los conocimientos ancestrales de la comunidad (FEDEOREWA, 2019). Los maestros afrodescendientes de la IE Katío Chamí también participan de la construcción de ese Tejido de Saberes, que busca establecer diálogos entre dos mundos, el mundo indígena y el mundo occidental. Al respecto, la maestra de bachillerato afirma lo siguiente:

Nosotros venimos de una parte occidental y hacemos un intercambio de cultura cuando entramos a un territorio diferente, o a una etnia diferente, que son los indígenas. A mí, como docente [afrodescendiente], me toca adaptarme al medio al cual estoy llegando, acoplarme. Mirar cuáles son sus costumbres, sus creencias, y de esa manera apropiarme del dialecto que ellos tienen (Entrevista, 31 de octubre de 2019).

Los Embera Chamí de la comunidad indígena de Sabaleta aún conservan su lengua nativa que, según el Gobernador, enseñan desde casa. Sin embargo, para este líder indígena es importante que los niños y niñas de la comunidad también aprendan el español en la escuela. Dicho en sus palabras:

Nosotros desde casa hablamos la lengua [Chamí], desde las escuelas hablamos el español, pero todo no puede ser español, por eso a nuestros muchachos se les dificulta la educación [occidental], va lento, ha sido lento. Igual, la idea es no recargar mucho

${ }^{22}$ Entrevista, 24 de octubre de 2019

${ }^{23}$ Entrevista, 31 de octubre de 2019 
Charry, O., Tamayo, C., \& Jaramillo, D. (2020). ¿Matemáticas para todos? Deconstrucciones desde un territorio chocoano. Revista Latinoamericana de Etnomatemática, 13(4), 95-119. DOI:

10.22267/relatem.20134.74

hacia lo occidental, ni tampoco mucho a [lo nuestro] porque ellos deben aprender [también lo del otro]. (Entrevista, 24 de octubre de 2019).

En la lengua Chamí, por ejemplo, al mirar en el diccionario Chamí de la comunidad indígena de Sabaleta, encontramos que la palabra matemáticas no existe; existen, sí, algunas palabras que denotan sus prácticas sociales, como, por ejemplo: sembrar (udai), recoger (pese), o bailar (koboabadai).

En una perspectiva wittgensteiniana, podríamos decir, apoyados en las ideas de Miguel (2016), que participar en un juego de lenguaje como, por ejemplo, la práctica social de sembrar (udai), de recoger (pese), o de bailar (koboabadai), también es hacer [matemáticas]. Pues como dice Wittgenstein (1987): “[...] si la matemática es un juego, entonces jugar un juego es hacer matemática, ¿y por qué no también bailar?” (Parte V, §4). Podríamos decir también, que la práctica social de bailar (koboabadai en Chamí) y la práctica social de hacer [matemáticas], son juegos de lenguaje normativos que mantienen entre sí semejanzas de familia.

Al emplear la noción semejanzas de familia, no sólo pretendemos mostrar, como lo señaló Condé (2004), que los juegos de lenguaje no guardan entre sí una esencia metafísica, sino también comprender su diferencia a partir de la propia noción de semejanza. Pues, como dice Condé (2004):

Wittgenstein comprende las semejanzas de familia a partir de la diferencia, es decir, al establecer esa analogía entre diversas características en el interior de un juego de lenguaje o entre varios juegos, [...] [Wittgenstein] no está propiamente buscando la identidad, la igualdad de un juego para otro, sino la diferencia que, a pesar de existir, todavía permite comprender aquella actividad como un juego de lenguaje en el interior del cual los usos de las palabras establecen las significaciones. En otros términos, como una semejanza de familia posibilita analogías, ella también permite percibir las diferencias. (p. 57).

En ese sentido, consideramos que las nociones de juego de lenguaje y semejanzas de familia propuestas por Wittgenstein, posibilitarían que viéramos el enunciado "matemáticas para todos" de otras maneras. Pues, ya no estaríamos hablando de esa imagen disciplinar de la matemática, sino de las [matemáticas] entendidas como prácticas sociales o juegos de lenguaje normativos. Y podríamos decir que existen tantas [matemáticas] como prácticas sociales; todas ellas emparentadas entre sí, sin que exista algo esencial que las atraviesa. Lo único que podríamos decir que hay entre ellas son semejanzas de familia. 
A partir de esas nociones de juego de lenguaje y semejanzas de familia, podríamos decir, como lo mostró Tamayo (2017), que las prácticas sociales de medir, contar o calcular, aunque presentan semejanzas o parecidos con las de la matemática escolar, ellas son diferentes. Se diferencian, por ejemplo, en los usos que hacemos de las palabras en esos juegos de lenguaje.

Según Condé (2004), el significado de las palabras está determinado por el uso que hacemos de ellas en el lenguaje cotidiano. En términos de este autor, contextos y "situaciones [distintas] pueden generar significaciones diferentes para la misma palabra” (p. 48). Por ejemplo, al hablar con el Rector de la IE Katío Chamí sobre algunas prácticas tradicionales de medición en las comunidades afrodescendientes del Chocó, encontramos que la palabra arroba tiene significados diferentes para la cultura occidental y la cultura afrodescendiente del Chocó. Cuenta el Rector de la IE que cuando uno va a la plaza a comprar pescado,

la arroba de pescado son 50 pescados apenas, y una arroba [en el sistema occidental] es 25 libras (...). Hay veces que da más de 100 libras. No depende de lo que pesa, sino de la [cantidad]. No miran cuánto pesa la arroba de pescado, sino [que cuentan] 50 pescados, pese lo que pese. (Entrevista, 14 de noviembre de 2019).

Otro ejemplo lo podemos encontrar en las prácticas de conteo, donde se movilizan diferentes usos de la palabra número. En el diccionario Chamí de la comunidad de Sabaleta, se encuentran los números del 1 al 5. Estos números se dirían así en lengua Chamí: uno (aba), dos (ôme), tres (ômbêa), cuatro (kîmârê) y cinco (juaâbâ). Para el maestro indígena Abelardo Tascón ${ }^{24}$ Embera Chamí de Valparaíso (Antioquia, Colombia), los números para su comunidad están relacionados con el entorno. Según él,

En el entorno, eso significa que tiene un comienzo y vuelve otra vez a él. Cuando terminamos en el número cinco significa que empieza una nueva era, que es nueva era para nosotros (...). Mire, es como con la muerte de un ser: o sea, yo me muero, pero nace alguien, otra persona, y esa es la relación de nosotros con el significado de los números. (Higuita, 2014, p. 168).

De acuerdo con Higuita (2014), el significado de cada uno de esos números para esa comunidad es el siguiente: uno, significa hermano; dos, significa camino; tres, limpieza; cuatro, unión de parejas; y, cinco, significa cambio de generación. Según esta misma autora,

\footnotetext{
${ }^{24}$ Maestro de la comunidad Embera Chamí de Valparaíso (Antioquia, Colombia). Su voz es traída a partir de la tesis de maestría de Higuita (2014).
} 
Charry, O., Tamayo, C., \& Jaramillo, D. (2020). ¿Matemáticas para todos? Deconstrucciones desde un territorio chocoano. Revista Latinoamericana de Etnomatemática, 13(4), 95-119. DOI:

$10.22267 /$ relatem.20134.74

el seis lo "escribían jua ą̧a ąba, que representa una mano y un hermano. Y así para los números hasta el 9. El número 10 se escribiría jua, el 20 ẽbẽrã ą̧a, el 20 que representaba el cuerpo de un Embera” (p. 169). Los números después del 20 no existen para esta comunidad, aunque se dicen en español por la necesidad del relacionamiento con las culturas no indígenas (Higuita, 2014).

Al mirar el significado de los números en la comunidad Gunadule de Necoclí (Antioquia), a través de la voz del líder indígena Green (2011), vemos que su significado está ligado estrechamente "al lenguaje; él permite expresar el tiempo, el espacio con sentido y coherencia, además da cuenta de las formas de vida, las reglas y las concepciones que la comunidad tiene acerca de la normatividad del saber moral” (Green, 2011, p. 167).

Según este autor, el número uno significa "madre (ser único), (...) [que] denota que la madre es irrepetible, irreemplazable, venimos de los huesos de ella, pertenecemos a ella, somos hueso de ella" (p. 168). El número dos significa

la paz del corazón, (...) [les] enseña el estado de armonía, felicidad, acogida, tranquilidad, madurez y responsabilidad al que llegan dos corazones que se han unido para amar, para ser una sola persona, conformando un sólo corazón para perpetuar la felicidad de la familia. (Green, 2011, p. 168).

El tres significa "el corazón de la abstinencia (...), significa la venida de un hijo o una hija (...)” (Green, 2011, p. 169). Este número "da a entender entonces la responsabilidad que implica para la pareja la gestación de un nuevo ser; para ambos este período significa abstinencia, cambio, responsabilidad y sintonía con la Madre Naturaleza (...)” (Ibídem, p. 170). Continuado con este autor, el número cuatro significa "doble abstinencia", que hace referencia a las autoridades tradicionales que debe tener la comunidad. El cinco significa “arpón (abuelo sabio)"; este número se compara con el cuerpo humano que también tiene cinco extremidades, las cuales son utilizadas para realizar diversas actividades (Green, 2011). También, según este autor, se compara el arpón, que es un instrumento de pesca, con un abuelo sabio que consigue la comida para poder alimentar a su familia y a la comunidad.

En ese sentido, como afirma Condé (2004), los significados surgen del uso que hacemos de las palabras en diferentes juegos de lenguaje; significados que están mediados por reglas, que constituirían la gramática. En esa perspectiva, apoyados en Tamayo (2017), consideramos que las matemáticas escolares, en contextos indígenas como los mencionados 
anteriormente, no describen las prácticas sociales de esas comunidades. Como vimos en el caso de la práctica de conteo, el uso de las palabras número, uno, dos, etc., describen las cualidades de ciertos objetos, que denotan las formas de conocer el mundo de esas comunidades (cosmovisiones), de manera diferente a como se entienden esas palabras en la práctica de conteo de la matemática escolar, que "se refiere a la acción de contar el número de elementos de un conjunto de objetos” (Tamayo, 2017, p. 243). Así, continuando con esta misma autora, el uso de esas palabras en la práctica de conteo, "sigue las reglas de la gramática de su cultura y no las de la gramática de la práctica de conteo de la matemática escolar" (Tamayo, 2017, p. 243).

Con relación a los cálculos mentales que realizaban tradicionalmente en la comunidad de Sabaleta con los números, el maestro de primaria dice lo siguiente:

Todos [nosotros] somos de pura oralidad. Nos falta es escribir (...). [Por ejemplo, nuestros ancestros, para hacer cálculos,] cogían las fases de la luna. La fase de la luna son treinta días, [es decir], un mes. Dos lunas serían dos meses, serían sesenta, (...), [y, así sucesivamente] (Encuentro reflexivo, 5 de noviembre de 2019).

De acuerdo con Knijnik (2006), las contribuciones de Ludwig Wittgenstein nos pueden aportar a nuestra comprensión sobre la oralidad. Por ejemplo, con relación a los cálculos de memoria, Wittgenstein (1995) dice lo siguiente:

Una persona hace una cuenta mentalmente. El resultado lo aplica, digamos, en la construcción de un puente o de una máquina. - ¿Quieres tú decir que ella [la persona] no encontró realmente este número por medio del cálculo? ¿Qué le cayó en el regazo, como en una especie de sueño? Tiene que haber habido cálculo y hubo cálculo, porque ella sabe que efectuó el cálculo y cómo lo efectuó; y el resultado correcto sería inexplicable sin cálculo. $(\S 364)$

Más adelante el filósofo compara el cálculo de memoria con el cálculo en el papel mostrando que entre ellos existe un parentesco, así: “«Efectué la multiplicación...mentalmente» — ¿No crees en tal afirmación? - ¿Pero fue realmente una multiplicación? No fue apenas «una» multiplicación, pero sí esta — efectuada en la cabeza. (...). «Este proceso mental corresponde a este proceso en el papel»” (§ 366).

De acuerdo con Knijnik (2006), Ludwig Wittgenstein nos muestra que el cálculo de memoria y el cálculo en el papel presentan semejanzas, esto es, habría entre ellos una semejanza de familia. Y, aunque entre esos dos tipos de cálculo existiría una correspondencia, esta no se 
Charry, O., Tamayo, C., \& Jaramillo, D. (2020). ¿Matemáticas para todos? Deconstrucciones desde un territorio chocoano. Revista Latinoamericana de Etnomatemática, 13(4), 95-119. DOI:

$10.22267 /$ relatem.20134.74

establece, según Knijnik (2006), “simplemente con la identificación del modo de operar mentalmente con los procedimientos escritos de los algoritmos" (p. 161).

De manera semejante, Wittgenstein (1987) compara aprender a calcular con la [aritmética], para mostrarnos que también ellos se encuentran emparentados, es decir, que presentan una semejanza de familia. El filósofo después de presentar un ejemplo donde cierta genta compra y vende leña, luego mide con un patrón los motones de leña, y para calcular el número de monedas que ha de darle a cada uno, multiplica las medidas de la anchura, longitud y altura, pregunta lo siguiente:

¿Quién calcula así tiene que expresar una 'proposición aritmética'? Es verdad que enseñamos a los niños la tabla de multiplicar en forma de frasecitas, pero ¿es eso esencial? ¿Por qué no habrían simplemente de: aprender a calcular? Y si saben hacerlo, ¿no han aprendido aritmética? (Parte I, §144).

A partir de las contribuciones de este filósofo, podríamos decir que las prácticas de calcular o contar en la forma como lo hacen en la comunidad indígena de Sabaleta también es hacer [aritmética]. Y que sus conocimientos orales también son [matemáticas]. Desde esta perspectiva, el enunciado "[matemáticas] para todos" en el marco del currículo [de matemáticas] de la IE Katío Chamí se vería de otra manera; como una agenda política de lucha y de resistencia por la preservación de los conocimientos ancestrales de la comunidad de Sabaleta.

\section{A MODO DE CIERRE}

En este artículo nos propusimos problematizar el enunciado "matemáticas para todos", presente en algunas reformas educativas desde la década del 90 en Colombia. Para ello, nos apoyamos en una actitud terapéutico deconstruccionista inspirada en las formas de pensar y practicar la filosofía de Ludwig Wittgenstein y Jacques Derrida. Desde esa perspectiva, no pretendimos negar ese enunciado, ni ponernos a favor o en contra de él. Sólo buscamos tratar de verlo de otras maneras, tener una visión panorámica de ese enunciado.

A través de las voces de los maestros y maestras de la IE Katío Chamí, en interlocución con voces de otros maestros y líderes indígenas colombianos, identificamos el carácter problemático que mantiene ese enunciado cuando alimentamos, en términos curriculares, una imagen disciplinar de la matemática. Es decir, cuando entendemos la matemática como un 
cuerpo jerarquizado, sistematizado y unificado de conocimientos abstractos que son independientes de las prácticas sociales.

Apoyados en la perspectiva política cultural de la educación matemática (Valero, 2018), en interlocución con autores como Veiga-Neto (2001) y Veiga-Neto y Lopes (2011; 2012), que fundamentan sus estudios sobre el currículo en las teorizaciones foucaultianas, intentamos desnaturalizar el enunciado "matemáticas para todos". En otras palabras, buscamos mostrar que ese enunciado no es natural ni inevitable, sino que es una construcción discursiva que promueve simultáneamente procesos de inclusión y exclusión de individuos, pueblos y naciones de acuerdo con su logro en matemáticas.

Al mirar las matemáticas desde una perspectiva indisciplinar, apoyados en las contribuciones del filósofo Ludwig Wittgenstein, logramos ver el enunciado "matemáticas para todos" de otras maneras. Ya no como una agenda política que promueve la homogeneización de la sociedad, sino como una agenda política de lucha y de resistencia por la preservación de diferentes formas de vida, por la recuperación de aquellos sujetos que han sido históricamente excluidos a través de relaciones asimétricas de poder, por ejemplo, indígenas.

\section{REFERENCIAS}

Charry, O. \& Jaramillo, D. (2020). Currículo de matemáticas y desarrollo en debate: reflexiones desde un territorio chocoano. REMATEC, 15(33), 31-52.

Charry, O., Jaramillo, D. \& Tamayo, C. (2020). Currículo de matemáticas: una problematización de la dicotomía teoría/práctica desde un territorio chocoano. Revista Educação \& Realidade, 45(3), e106760. http://dx.doi.org/10.1590/21756236106760.

Charry, O., Tamayo, C. \& Jaramillo, D. (2020). "Indisciplina" en Chocó: una problematización de las prácticas disciplinarias en tiempos de pandemia. Revista Latinoamericana de Etnomatemática, 13(1), 125-149.

Condé, M. (2004). As Teias da Razão: Wittgenstein e a crise da racionalidade moderna. Belo Horizonte: Argumentum.

Cuellar, R. (2017). Nabba nana gala burbaba nanaedi igala odurdagge gunadule durdagedi nega gine: igal dummadi maidi sabbimala soganergwa naggulemaladi. (En español: La pedagogía de la Madre Tierra en una escuela indígena Gunadule: un estudio sobre la sabiduría de seis plantas de protección). (Tesis de maestría). Universidad de Antioquia, Medellín.

D'Ambrosio, U. (2008). Etnomatemática: Eslabón entre las tradiciones y la modernidad. México: Limusa. 
Charry, O., Tamayo, C., \& Jaramillo, D. (2020). ¿Matemáticas para todos? Deconstrucciones desde un territorio chocoano. Revista Latinoamericana de Etnomatemática, 13(4), 95-119. DOI:

10.22267/relatem.20134.74

Derrida, J. \& Roudinesco, E. (2004). Y mañana qué. México: Fondo de Cultura Económica.

Foucault, M. (2002). Vigilar y Castigar; Nacimiento de la Prisión. 1ª ed. Buenos Aires: Siglo XXI Editores.

Glock, H. (1997). Dicionário Wittgenstein. Rio de Janeiro: Zahar.

Green, S. (2011). Significados de vida: espejo de nuestra memoria en defensa de la madre Tierra (Anmal Gaya Burba: isbeyobi daglege nana nabgwana bendaggegala). (Tesis de doctorado). Universidad de Antioquia, Medellín.

Cáisamo, I. (2012). Pensar bien el camino de la sabiduría= Kirincia bio o kuitá. (Tesis de doctorado). Universidad de Antioquia, Medellín.

Higuita, C. (2014). La movilización de objetos culturales desde las memorias de la práctica de construcción de la vivienda tradicional Embera Chamí: posibilidades para pensar el (por)venir de la educación (matemática) indígena (Tesis de Maestría). Universidad de Antioquia, Medellín.

PEC. (2015). Proyecto Educativo Comunitario de la Institución Educativa Katio Chamí. (Documento inédito). Sabaleta: El Carmen de Atrato, Chocó.

Knijnik, G. (2006). La oralidad y la escritura en la educación matemática: reflexiones sobre el tema. Educación matemática, 18(2), 149-165.

Lizcano, E. (1992). De Foucault a Serres: notas para una arqueología de las matemáticas. Theoria. Revista de Teoría, Historia y Fundamentos de la Ciencia, 7(1/2/3), 499507.

Lizcano, E. (2006). Metáforas que nos piensan: sobre ciencia, democracia y otras poderosas ficciones. Madrid: Traficantes de Sueños.

Mecha, B. (2007). Una mirada de emberá sobre el conocimiento y la investigación. Revista Educación y Pedagogía, 19(49), 103-118.

Mecha, B. (2015) La Unidad Entre La Diversidad Natural Y Cultural Como Herramienta Metodológica. (Documento inédito). Chocó: Quibdó.

Mejía, M. (2004). Leyendo las políticas educativas de la globalización. En M. R. Mejía, Pensamientos y realidades de la educación contemporánea, 8-31.

Miguel, A. (2015). A Terapia Gramatical-Desconstrucionista como Atitude de Pesquisa (Historiográfica) em Educação (Matemática). Perspectivas da Educação Matemática, 8(18), 607-647.

Miguel, A. (2016). Entre Jogos de Luzes e de Sombras: uma agenda contemporânea para a educação matemática brasileira. Perspectivas da Educação Matemática, 9(20).

Monteiro, A. (2005). Currículo de matemática: reflexões numa perspectiva Etnomatemática. $7^{\circ}$ Encuentro de Educación Matemática, Asocolme, Tunja.

OREWA (2010). Lo que queremos y pensamos hacer en nuestro territorio. Quibdó: Asociación Orewa (OREWA). 
Quiceno, A. \& Montoya, D. (2020). matemáticaS, educación y paz en la escuela (Tesis de maestría). Universidad de Antioquia, Medellín.

Santos, B. (2008). Un discurso sobre las ciencias. In: B. Sousa (ed.). Una epistemología del sur: la reinvención del conocimiento y la emancipación. México DF: Siglo XXICLACSO.

Santos, B. (2019) El Fin del Imperio Cognitivo: la afirmación de las epistemologías del sur (A. Torradellas Trad.) Madrid: Editorial Trotta.

Skliar, C. (2005). Derrida \& a educação. 1. ed. Belo Horizonte: Autêntica.

Souza, E., \& Miguel, A. (2020). A Encenação de Práticas Culturais na Tessitura de Outras Escolas: a vida como eixo da ação educativa. REMATEC, 15(33), 166-184.

Tamayo, C. (2017). Vení, vamos hamacar el mundo, hasta que te asustes: uma terapia do desejo de escolarização moderna (PhD Thesis). Universidade Estadual de Campinas, São Paulo.

Tamayo, C., \& Cuellar, R. N. (2016). Juegos de lenguaje en movimiento: Una experiencia Indígena. Revista Latinoamericana de Etnomatemática, 9(1), 49-70.

Valero, P. (2017a). El deseo de acceso y equidad en la educación matemática. Revista Colombiana de Educación, (73), 99-128.

Valero, P. (2017b). Mathematics for All, Economic Growth, and the Making of the CitizenWorker. In T.S. Popkewitz, J. Díaz \& C. Kirchgasler (Eds.), A political sociology of educational knowledge: Studies of exclusions and difference, 117-132.

Valero, P. (2018). Capital humano: O currículo de matemática escolar e a fabricação do homus oeconomicus neoliberal. In E. V. Godoy, M. A. da Silva, \& V. d. M. Santos (Eds.), Currículos de matemática em debate: Questões para políticas educacionais e para a pesquisa em Educação Matemática, 43-68.

Valero, P. \& García, G. (2014). El Currículo de las Matemáticas Escolares y el Gobierno del Sujeto Moderno. Boletim de Educação Matemática 49(28), 491-515. Recuperado de https://www.redalyc.org/pdf/2912/291231725003.pdf

Valero, P. \& Knijnik, G. (2015). Governing the modern, neoliberal child through ict research in mathematics education. In For the Learning of Mathematics, 35(2), 33-38.

Valero, P., Andrade-Molina, M. \& Montecino, A. (2015). Lo político en la Educación Matemática: de la Educación Matemática Crítica a la Política Cultural de la Educación Matemática. Revista Latinoamericana de Matemática Educativa, 18(3), 287-300.

Veiga-Neto, A. (2001). Incluir para saber. Saber para excluir. Pro-posições, 12(2-3), 22-31.

Veiga-Neto, A., \& Lopes, M. (2011). Gubernamentalidad, biopolítica e inclusión. In: SALCEDO, R. A. C.; DÍAZ, D. L. M. (Org.). Gubernamentalidad y Educación: discusiones contemporáneas, 105-126. 
Charry, O., Tamayo, C., \& Jaramillo, D. (2020). ¿Matemáticas para todos? Deconstrucciones desde un territorio chocoano. Revista Latinoamericana de Etnomatemática, 13(4), 95-119. DOI:

10.22267/relatem.20134.74

Veiga-Neto, A., \& Lopes, M. (2012). La inclusión como dominación del otro por él mismo. Pedagogía y saberes, (36), 57-68.

Vilela, D. (2010). A Terapia filosófica de Wittgenstein e a educação matemática. Educação e Filosofia Uberlándia, 24(48), 435-456.

Wittgenstein, L. (1987). Observaciones sobre los Fundamentos de la Matemática. Traducción de A. G. Suárez; U. Moulines. Madrid: Alianza.

Wittgenstein, L. (1995). Tratado Lógico-Filosófico * Investigacoes Filosóficas (M.S. Lourenço Trad., 2 ed.). Lisboa: Fundação Calouste Gulbenkian. (Originals, 1961 y1985, respectively).

Wittgenstein, L. (2007). Observaciones Sobre o Ramo Dourado de Frazer. Almeida, J. (Trad.). Almeida, J. J. A. Em Suplemento da Revista Digital AdVerbum, v. 2, 186231. 